\title{
Die Berliner SDG Sommerakademie 2018: Leave No One Behind! Zivilgesellschaftliche Beteiligung im Kontext der Agenda 2030
}

\begin{abstract}
"Ich konnte sehen, dass es möglich ist mich für die Welt zu engagieren. Ich brauche nur Wille, Organisation und Menschen, die das gleiche Ideal haben. "So lautet das Fazit einer Teilnehmerin der SDG Sommerakademie, die vom 4. bis 7. September 2018 in Berlin stattfand. Das EPIZ (Zentrum für Globales Lernen in Berlin e.V.) und die Berliner Außenstelle von Engagement Global gGmbH hatten Studierende unterschiedlicher Fächer und Referent/inn/en aus Theorie und Praxis eingeladen, um über Fragen Zivilgesellschaftlichen Engagements im Rahmen der Agenda 2030 zu diskutieren, Erfahrungen auszutauschen, Beispiele guter Praxis im Globalen Norden und Süden kennen zu lernen und die eigene Handlungskompetenz zu erweitern.
\end{abstract}

Es war bereits die dritte Sommerakademie für Studierende zum Thema Nachhaltige Entwicklungsziele und Agenda 2030, die seit 2016, ein Jahr nach deren Verabschiedung, angeboten wurde. Während es in den beiden Jahren zuvor darum ging, jeweils ein Sustainable Development Goal (SDG) vorzustellen - 2016 das Thema „Nachhaltige(r) Konsum und Produktion“ (SDG 12) und 2017 „Frieden, Gerechtigkeit und Inklusion“ (SDG 12) hatten die Veranstalterinnen in diesem Jahr ein Thema gewählt, das alle Entwicklungsziele in den Blick nehmen sollte.

Die 17 Ziele der Agenda sind getragen von der Idee einer gemeinsamen Verantwortung aller für die Menschen und den Planeten und legen den Fokus auf die Themenbereiche „Menschen“, „Planet und Umwelt", „Wohlstand“, „Frieden“ und „Partnerschaft", die im Sinne einer ökonomischen, ökologischen und sozialen Entwicklung in Einklang gebracht werden müssen. Die Verantwortung aller an der Verwirklichung der Ziele im globalen Norden und Süden ist zentrales Anliegen der Agenda 2030 und so auch die Teilhabe aller an Entscheidungs- und Umsetzungsprozessen. Besonders der Aspekt der Inklusion aller ist das Herzstück der Agenda mit dem Leitsatz „Leave No One Behind“.

Mit diesem Thema waren unterschiedliche Ebenen zivilgesellschaftlicher Teilhabe für den Agenda 2030-Prozess angesprochen, die im Rahmen der Akademie berücksichtigt werdensollten. Zuersteinmal mussteesumeineBegriffsbestimmung gehen. Was meinen wir mit den Begriffen Partizipation und Beteiligung? Wann können wir von der Teilhabe aller sprechen

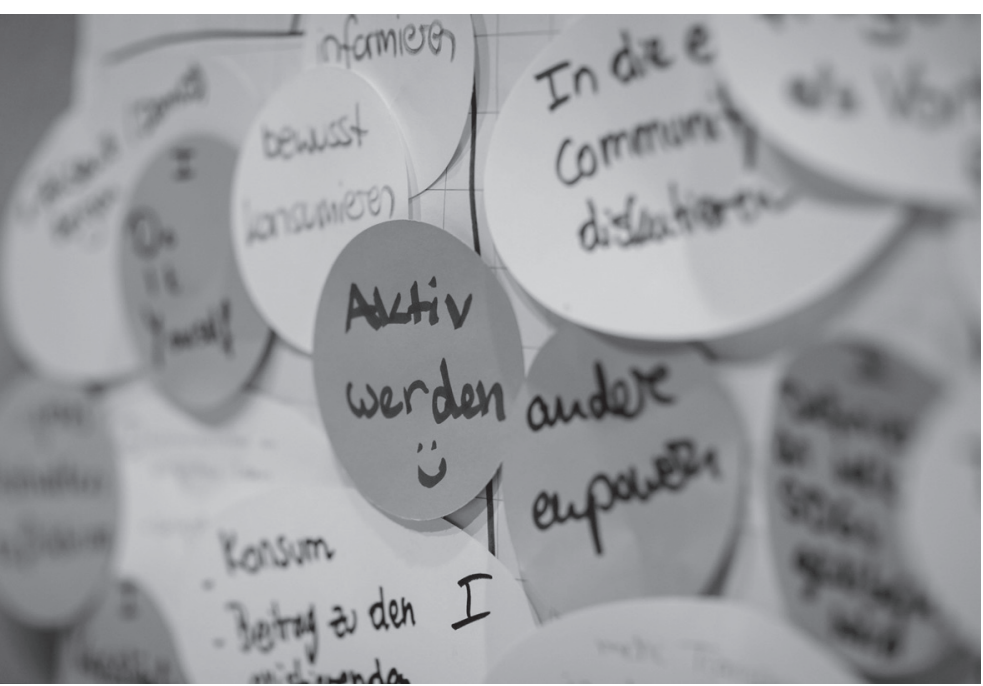

Abb. 1: „Aktiv werden“, Quelle: Janin Schmitz (EPIZ Berlin)

und wann nicht? Und wer ist eigentlich „die Zivilgesellschaft“?

In einem einführenden Beitrag vermittelte Iven Saadi am Beispiel der Kinderrechte und Teilhabe von Kindern und Jugendlichen, was Partizipation sein kann und warum es Erwachsenen so schwer fällt, Kindern und Jugendlichen die Teilhabe an den gesellschaftlichen und politischen Prozessen, die sie betreffen, einzuräumen.

Auch ging es um die Frage, welche Inhalte und Ziele zivilgesellschaftliche Akteure in der Formulierung und Umsetzung der Agenda 2030 in ihrem Entstehungsprozess und aktuell bei der nationalen Umsetzung der Ziele einbringen können. Von Beginn an war der Prozess der Auswahl und Formulierung der SDGs durch einen Multistakeholder-Prozess gedacht und gewollt. Marie-Luise Abshagen berichtete von ihren Erfahrungen als Vertreterin des Forums für Umwelt und Entwicklung und darüber, wie die Teilhabe auf internationaler Ebene möglich war und welche Hindernisse sie in dem Prozess Anliegen unterzubringen erlebt hat. Den Blick auf die nationale Umsetzung ermöglichte Roman Fleißner, Referent für Globales Lernen und Verantwortlicher für die Umsetzung des Agenda Prozesses bei der AWO International, der die aktuelle Arbeit in Deutschland vorstellte. Die Umsetzung der SDGs in Deutschland ist bisher zwar in einem nationalen Aktionsplan formuliert, allerdings wenig bekannt. Besonders diese beiden 
Berichte aus der Praxis zivilgesellschaftlicher Vertrter/-innen machten den Teilnehmer/inne/n die Chancen und Grenzen der Einflussnahme und auch die Konkurrenz und fehlende Kohärenz der unterschiedlichen Interessenvertreter/-innen auf internationaler und nationaler Ebene deutlich.

Besonders wichtig war es im Programm der Sommerakademie, Studierenden einen offenen Raum zu lassen, in dem sie eigene Themen und Fragen mit Anderen bearbeiten konnten. Hier ging es u.a. um die Vermittlung von Bildungsinhalten, wie Deep Democracy oder die wirkungsvolle Planung und Umsetzung von eigenen Aktionen mit anderen Aktiven.

Vertreter/-innen zivilgesellschaftlicher Organisationen, wie Silas Kropf von Amaro Drom e.V., Narcisse Djakam von Integritude e.V., Sabine Schielmann und Pedro Cona Caniullan des Instituts für Ökologie und Aktionsethnologie e.V. (Infoe) beleuchteten anhand ihrer Praxis, wie benachteiligte Gruppen im Globalen Norden und Süden mehr Teilhabe einfordern.

Daran anschließend sprach Fabian Kursawe von mohio e.V. zur Frage, wie nachhaltiges Engagement und die Beteiligung an politischen und gesellschaftlichen Prozessen gelingen kann und wie wir von den guten Vorsätzen zu einer guten Umsetzung kommen. In unterschiedlichen Workshops erweiterten die Teilnehmer/-innen ihre Handlungskompetenz. So wurden Workshops zur Planung von Kampagnen und Aktionen (Inkota e.V.) angeboten, Christina Nauditt stellte die Zukunftswerk- statt als Methode in politischen Planungsprozessen vor und Gabriela Turano übte Handlungs- und Lösungsmöglichkeiten für Konflikte und Formen von Unterdrückung durch das Forum Theater.

Zum Thema Partizipation und Medien berichtete die Journalistin Joanna Maria Stolarek von den Neuen Deutschen Medienmachern, die einen Beitrag zu mehr Vielfalt in der deutschen Medienlandschaft leisten und durch Materialien, Kampagnen und Schulungen zu diskriminierungssensiblem Umgang mit Sprache in den Medien arbeiten. Simon Didszuweit und Judith Liesenfeld von der Deutschen Welle Akademie stellten ihre internationale Arbeit, wie z.B. die Speak Up Kampagne, ein Beitrag zu mehr Teilhabe in Medien in Ländern des Südens, vor.

Natürlich waren vier Tage nicht genug, um die Bandbreite des Themas vollständig zu erfassen und alle Fragen zu beantworten. Dennoch wurde in den Rückmeldungen der Teilnehmer/-innen deutlich, dass sie ihr Wissen und ihre Fähigkeiten rund um das Thema Teilhabe und SDGs erweitern konnten und einen Raum des Austausches und der Begegnung hatten. Oder wie eine Teilnehmerin uns schrieb: „Toll, jeden Tag ein neues Puzzelstück, das hat etwas ins Rollen gebracht."

Christina Ayazi, EPIZ Berlin

ayazi@epiz-berlin.de

doi.org/10.31244/zep.2018.03.09

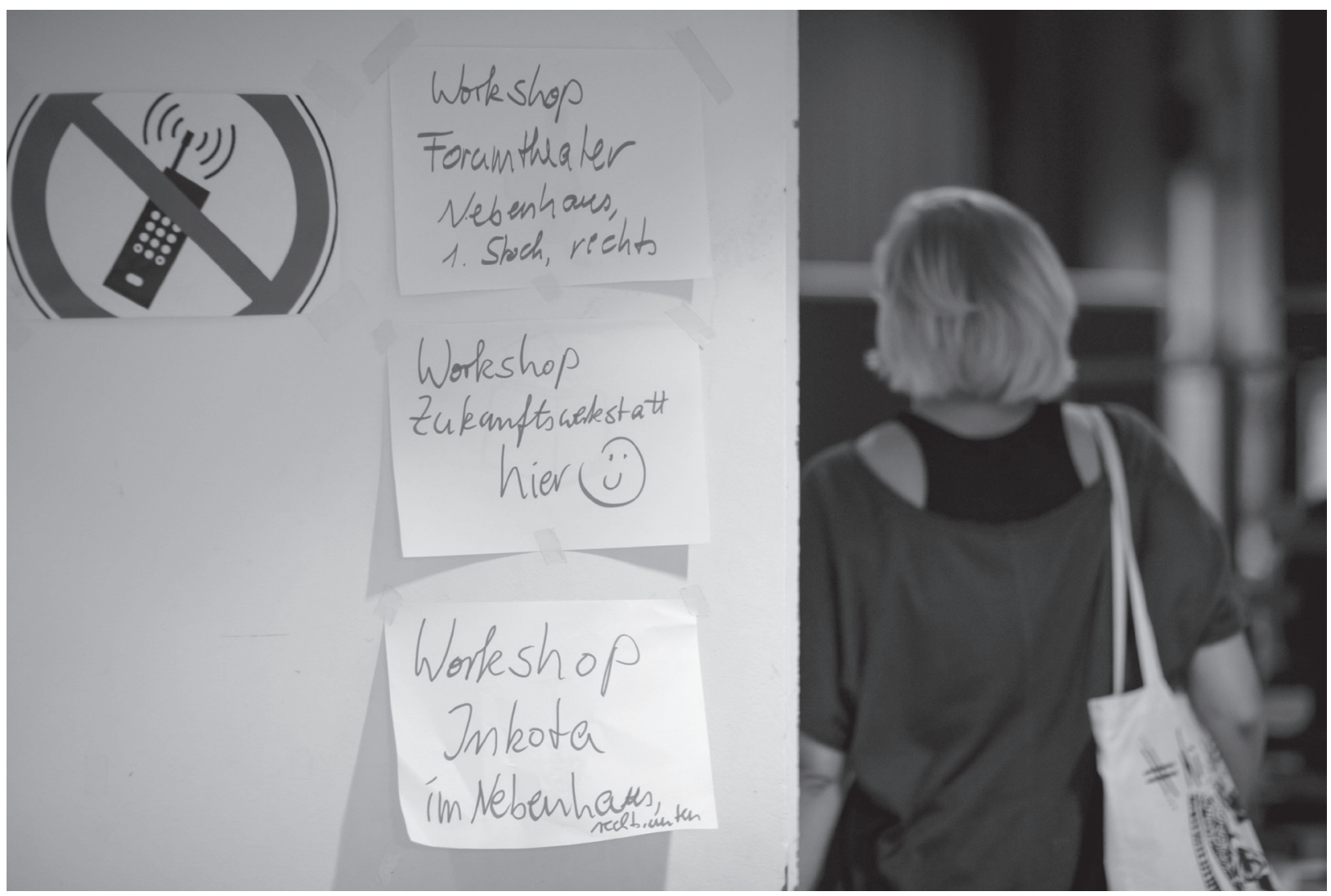

Abb. 2: „Workshops zu Partizipation", Quelle: Janin Schmitz (EPIZ Berlin) 